\title{
THE EFFECT OF ENVIRONMENT ON MILKY-WAY-MASS GALAXIES IN A CONSTRAINED SIMULATION OF THE LOCAL GROUP
}

\author{
Peter Creasey ${ }^{1}$, Cecilia Scannapieco ${ }^{1}$, Sebastián E. Nuza ${ }^{1}$, \\ Gustavo Yepes $^{2}$, Stefan Gottlöber ${ }^{1}$, and Matthias Steinmetz ${ }^{1}$ \\ ${ }^{1}$ Leibniz-Institut für Astrophysik Potsdam (AIP), An der Sternwarte 16, D-14482, Potsdam, Germany \\ ${ }^{2}$ Grupo de Astrofísica, Universidad Autónoma de Madrid, Madrid E-28049, Spain \\ Received 2014 November 20; accepted 2014 December 23; published 2015 February 4
}

\begin{abstract}
In this Letter, we present, for the first time, a study of star formation rate (SFR), gas fraction, and galaxy morphology of a constrained simulation of the Milky Way (MW) and Andromeda (M31) galaxies compared to other MW-mass galaxies. By combining with unconstrained simulations, we cover a sufficient volume to compare these galaxies' environmental densities ranging from the field to that of the Local Group (LG). This is particularly relevant as it has been shown that, quite generally, galaxy properties depend intimately upon their environment, most prominently when galaxies in clusters are compared to those in the field. For galaxies in loose groups such as the LG, however, environmental effects have been less clear. We consider the galaxy's environmental density in spheres of $1200 \mathrm{kpc}$ (comoving) and find that while environment does not appear to directly affect morphology, there is a positive trend with SFRs. This enhancement in star formation occurs systematically for galaxies in higher density environments, regardless whether they are part of the LG or in filaments. Our simulations suggest that the richer environment at megaparsec scales may help replenish the star-forming gas, allowing higher specific SFRs in galaxies such as the MW.
\end{abstract}

Key words: cosmology: theory - galaxies: evolution - galaxies: formation - methods: numerical

Supporting material: animation

\section{INTRODUCTION}

During the last decades, observational studies found indications that the environment where galaxies form plays an important role on the determination of their final properties. For example, elliptical galaxies are more strongly clustered than spirals (e.g., Dressler 1980; Hermit et al. 1996; Guzzo et al. 1997), and correlations between environment and the photometric color or luminosity of a galaxy have also been measured (Blanton et al. 2003). The suppression of star formation in clustered environments also extends to groups (Lewis et al. 2002; Coenda et al. 2014) and the morphology trends, i.e., that early-type galaxies lie closer to halo centers, extend as a continuum to group scales (Girardi et al. 2003). However, at stellar masses closer to that of the MW, this is more controversial (Ziparo et al. 2013) and some authors suggest large-scale features such as filaments may be more important than the group (e.g., Bahé et al. 2013). Group galaxies also appear deficient in cold gas (Hess \& Wilcots 2013), although McGee et al. (2008) find that while the disk fraction depends on the group environment, the other properties of the disks appear largely unaffected.

On the other hand, theoretical studies in the context of $\Lambda$ Cold Dark Matter $(\Lambda \mathrm{CDM})$ show that the merger rate of galaxies depends on environment, such that galaxies in intermediate- and high-density regions have the highest fractions of mergers (e.g., Maulbetsch et al. 2007; de Ravel et al. 2009; Darg et al. 2010; Ellison et al. 2010; Fakhouri et al. 2010; Lin et al. 2010; Jian et al. 2012; Tonnesen \& Cen 2012). Mergers are indeed known to induce morphological transformations of galaxies (e.g., Toomre \& Toomre 1972) and could be responsible for at least some of the observed trends of galaxy properties with environment.
Our MW lives in a rich group environment, known as the "Local Group" (LG). Andromeda (M31), the other large spiral in the LG, has a mass similar to the MW, and lies at less than a megaparsec away. Such spiral galaxies are prevalent in the universe, and thus a fundamental test of the $\Lambda \mathrm{CDM}$ cosmological framework is that neither the local universe (e.g., Nuza et al. 2014a) nor the MW and M31 should be extremely improbable objects.

The numerical simulation of MW-like galaxies is an active area of research, with zoom-in techniques (Katz \& White 1993) allowing the collisionless and collisional components of cosmological density fluctuations to be followed over almost the full 13.7 billion years of cosmic time. Modern computers enable simulations that describe the internal properties of galaxies so that time variations due to mergers, interactions, and infall can indeed be quantified using an acceptable number of resolution elements.

The precise initial conditions (ICs) to produce late-type galaxies such as the MW and M31 are not believed to be strict as spiral galaxies are the most abundant type in our universe. In most modern studies (e.g., Scannapieco et al. 2009; Guedes et al. 2011; Aumer et al. 2013; Stinson et al. 2013; Vogelsberger et al. 2014) the ICs are chosen as halos of MW mass and isolated from other massive halos that can destroy disks at late times. Indeed the primary uncertainty of such simulations lies in the unresolved baryonic physics that result in star formation and feedback (see Creasey et al. 2011), and different codes applied to the same halo can produce substantial variation in the stellar component (Scannapieco et al. 2012).

While the interaction of the M31-MW pair is not yet expected to affect internal galaxy properties as they are still too distant, their environments on megaparsec scales have merged, and this overdense environment may affect the pair. One method to test such effects is to identify analogous pairs 
produced by cosmological ICs and compare them to more isolated systems. Following this approach, Few et al. (2012) found that for simulated MW-mass galaxies the difference between the field and loose groups appears marginal, and in fact they detected no visible difference between MW-like field galaxies and those that reside in loose groups. GarrisonKimmel et al. (2014) find no difference in concentrations or stellar masses within the virial radius, but do find an increased number of "backsplash" galaxies (see, e.g., Knebe et al. 2011) - those that have escaped the virial radius-for pairs such as the MW and M31.

Although those simulations allow assessment of the effects of environment on the formation of MW-mass galaxies, they do not fully exploit the detailed constraints we have from the present-day dynamics of our local universe. One method to ameliorate this is to utilize the power of constrained ICs that reproduce the observed dynamical properties of the LG in combination with hydrodynamical simulations, which has not previously been attempted. This provides a pertinent test to quantify the influence of environment, as well as allowing us to discern directly the physical processes in play during the formation of galaxies in a LG-like environment.

In this Letter, we present results from a simulation constrained both to form a LG analogue and to match the velocity field of the local universe. This allows us to contrast the properties of the simulated LG galaxies with other galaxies of similar stellar mass. For our LG simulation we have taken (a version with slightly greater coverage of) the ICs used to produce a MW-M31 pair in Nuza et al. (2014b) and Scannapieco et al. (2015) in a volume resembling the distribution of matter of our local universe. To enlarge and contrast our sample, we include the eight galaxies of Scannapieco et al. (2009, S09 hereafter) which use the same code. The latter simulations are resimulations of the eight halos of the Aquarius Project (Springel et al. 2008) that result in isolated galaxies at $z=0$.

This Letter is organized as follows. In Section 2 we describe the simulation code that was used to evolve the ICs of both the LG and Aquarius, and the sample of MW-analogues that are produced. In Section 3 we analyze the evolution of the environment of these galaxies and attempt to discern its effect on basic galaxy quantities such as stellar mass, gas mass and star formation rate (SFR). In Section 4 we present discussions and conclusions.

\section{SIMULATIONS}

\subsection{Initial Conditions}

The ICs used for our LG simulations are part of the Constrained Local Universe Simulations ${ }^{3}$ project. The ICs reproduce, by construction, the known dynamical properties of our local environment at $z=0$ (Gottlöber et al. 2010; Yepes et al. 2014), and are consistent with a $\Lambda$ CDM universe with WMAP-5 parameters: $\Omega_{M}=0.279$ (matter density), $\Omega_{\Lambda}=0.721$ (dark energy density), $\Omega_{\text {bar }}=0.046$ (baryon density), $H_{0}=100 h \mathrm{~km} \mathrm{~s}^{-1} \mathrm{Mpc}^{-1}$ with $h=0.7$ (Hubble parameter), and $\sigma_{8}=0.8$ (normalization of the power spectrum).

The ICs use the zoom-in technique, where particles are placed in a periodic box of $91 \mathrm{Mpc}$ on a side and our high-

http://www.clues-project.org/ resolution region is of $10 \mathrm{Mpc}$ radius at $z=0$, with a mass resolution of $2.8 \times 10^{6} M_{\odot}$ and $5.6 \times 10^{5} M_{\odot}$ in dark matter and gas particles, respectively, and a gravitational softening length of $0.7 \mathrm{kpc}$, fixed in physical coordinates since $z=3$ and fixed in comoving coordinates at earlier times. The IC phases are the same as for Nuza et al. (2014b), but the zoom region is slightly larger, causing some variation in the simulated evolution.

The Aquarius simulations that we use to compare with our LG galaxies are the eight galaxies first presented in S09. These are the hydrodynamical counterparts of the galaxies of the Aquarius Project, selected to have formed in isolated environments by requiring that they have no neighbor exceeding half their mass within a sphere of $1.4 \mathrm{Mpc}$ at $z=0$. The cosmological parameters are slightly different than those used in our LG simulation: $\Omega_{M}=0.25, \Omega_{\Lambda}=0.75, \Omega_{\text {bar }}=0.04$, $h=0.73$, and $\sigma_{8}=0.9$. The mass resolution and softening lengths adopted are, however, similar in the two samples, and we have used the same set of input parameters for star formation and feedback. The different cosmological parameters used are not expected to compromise our results as the adjustment to the cosmology is rather minor in comparison with the dispersion in the evolutionary trends.

\subsection{Simulation Code}

We use an extended version of the Tree-PM Smoothed Particle Hydrodynamics code GADGET3 (Springel et al. 2008) that includes metal-dependent cooling, chemical enrichment, and feedback from Type II and Ia supernovae, a multiphase gas model, and a UV background field (Haardt \& Madau 1996). The model has been developed in (Scannapieco et al. 2005, 2006) and is the same code as for (Nuza et al. 2014b).

In previous work, we have shown that our model is able to reproduce the formation of galaxy disks from cosmological ICs (Scannapieco et al. 2008, 2009) and alleviates the angular momentum problem. Some limitations exist, for example, simulated galaxies tend to have overly massive bulges (though see the implementation of Aumer et al. 2013), where the bulge mass is significantly reduced via early stellar feedback). However, disks do have realistic sizes and angular momentum content (Scannapieco et al. 2008, 2009, 2010, 2012), allowing studies of their formation and evolution in relation to those structures on larger scales. In a companion paper (Scannapieco et al. 2015) we analyze the evolution of the distribution of the stellar component, to which we refer the reader for details of morphology classification.

\subsection{The Galaxy Sample}

We choose a sample of galaxies with stellar masses in the range $10^{10.5-11.5} M_{\odot}$, i.e., similar to the MW. For our LG simulation, this includes the M31 and MW candidates ${ }^{4} \mathrm{G} 1$ and G2 along with four other galaxies that we refer to as G3-G6. These galaxies have no "contamination" within 1200 comoving $\mathrm{kpc}$ (ckpc) from their centers, i.e., they are sufficiently embedded in the zoom region to avoid low-resolution particles. The environment of these galaxies in gas and star-forming gas is projected in Figure 1, along with insets to enlarge the properties within the virial radius. All the galaxies in the LG

\footnotetext{
$\overline{4}$ Note that in Nuza et al. (2014b) these galaxies were referred to as $\mathrm{M} 31^{\mathrm{c}}$ and $\mathrm{MW}^{\mathrm{c}}$, respectively.
} 


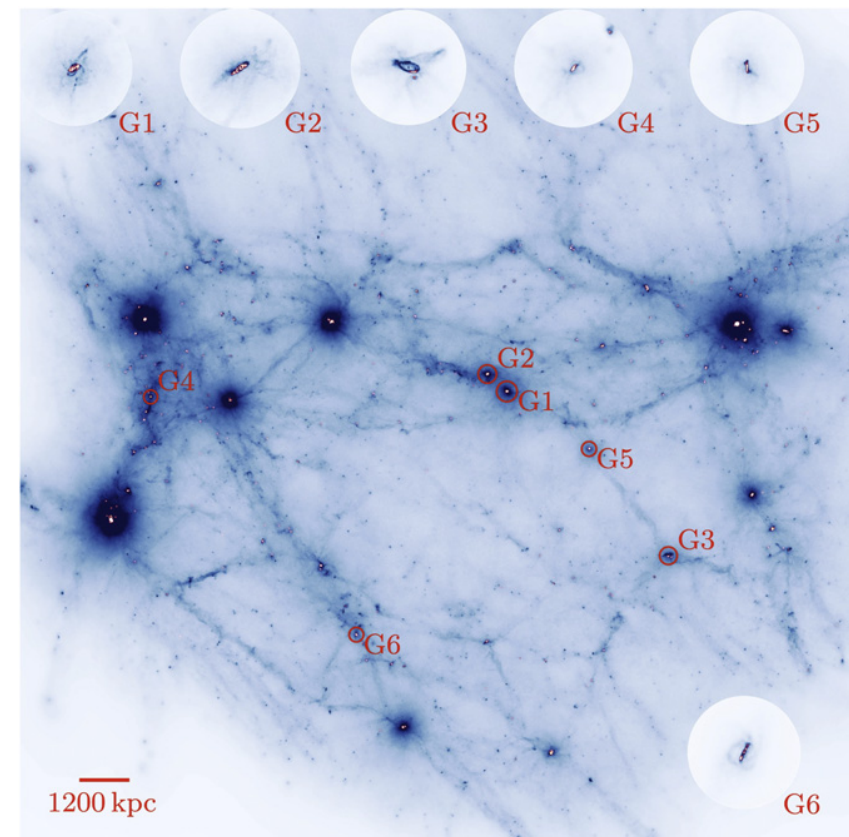

Figure 1. Column density of gas (blue) overlaid with star-forming gas (orange-white) for the zoom region at $z=0$. Red circles delimit the $R_{200}$ for each galaxy of a similar stellar mass to the MW (G1-G6), and the labeled insets enlarge these regions. $1200 \mathrm{kpc}$ is the radius of our environment measure, and the width of the image is $20 \mathrm{Mpc}$.

(An animation of this figure is available.)

Table 1

Main Properties of the LG and Aquarius Galaxies at $z=0$ : Virial Radius $\left(R_{200}\right)$ , Virial Mass $\left(M_{200}\right)$, and Masses in Stars, Gas, and Star-forming Gas $\left(M_{\text {star }}\right.$, $M_{\mathrm{gas}}$ and $M_{\mathrm{SF}}$, within the Virial Radius

\begin{tabular}{lccccc}
\hline \hline & $\begin{array}{c}R_{200} \\
(\mathrm{kpc})\end{array}$ & $\begin{array}{c}M_{200} \\
\left(10^{10} M_{\odot}\right)\end{array}$ & $\begin{array}{c}M_{\text {star }} \\
\left(10^{10} M_{\odot}\right)\end{array}$ & $\begin{array}{c}M_{\text {gas }} \\
\left(10^{10} M_{\odot}\right)\end{array}$ & $\begin{array}{c}M_{\mathrm{SF}} \\
\left(10^{10} M_{\odot}\right)\end{array}$ \\
\hline G1 & 245 & 168 & 8.1 & 6.6 & 0.479 \\
G2 & 219 & 120 & 6.4 & 5.2 & 0.369 \\
G3 & 211 & 108 & 6.8 & 3.6 & 0.078 \\
G4 & 166 & 52 & 3.3 & 1.8 & 0.146 \\
G5 & 177 & 63 & 4.4 & 1.8 & 0.151 \\
G6 & 174 & 60 & 3.7 & 1.5 & 0.090 \\
\hline Aq-A & 232 & 149 & 9.2 & 4.9 & 0.153 \\
Aq-B & 181 & 71 & 4.0 & 1.7 & 0.033 \\
Aq-C & 237 & 161 & 11.0 & 3.8 & 0.119 \\
Aq-D & 233 & 149 & 8.4 & 3.5 & 0.005 \\
Aq-E & 206 & 108 & 8.4 & 2.6 & 0.048 \\
Aq-F & 196 & 91 & 7.7 & 1.8 & 0.012 \\
Aq-G & 180 & 68 & 4.5 & 1.6 & 0.061 \\
Aq-H & 182 & 74 & 6.5 & 0.6 & 0.011 \\
\hline
\end{tabular}

sample are well separated ( $>3 \mathrm{Mpc})$, except the G1-G2 pair at $770 \mathrm{kpc}^{5}$.

The eight Aquarius galaxies (denoted AqA-H) inhabit disjoint environments and were resimulated individually. Images of these galaxies can be seen in S09 and for previous studies of their formation histories and properties, we refer the reader to (Scannapieco et al. 2009, 2010, 2011). Table 1

\footnotetext{
5 A visualization of a revolution of this structure and the environment selections is available at www.aip.de/en/research-area-ea/research-groups-andprojects/cosmology/galaxy-formation.
}

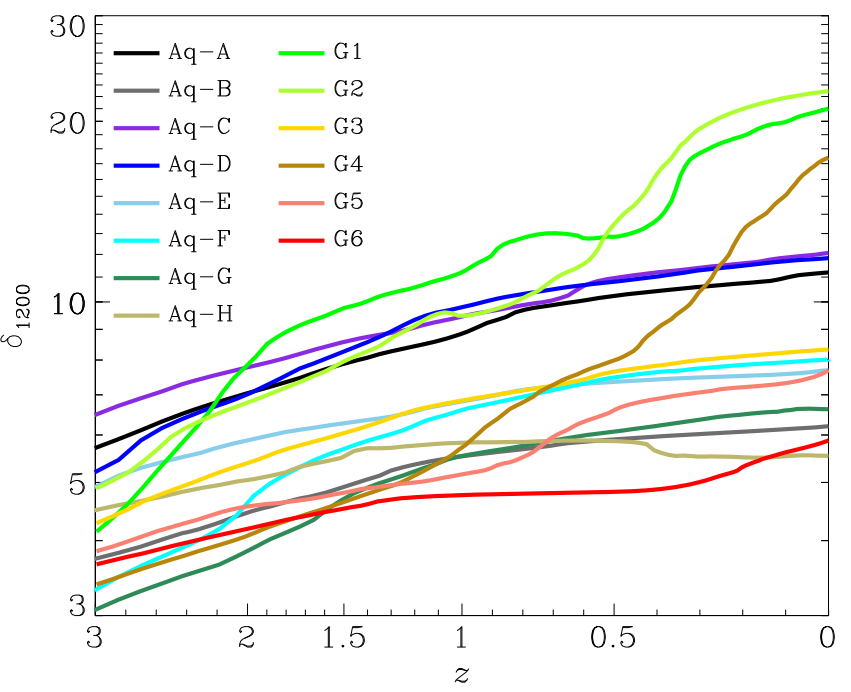

Figure 2. Environmental overdensity $\delta_{1200}$ vs. redshift for the galaxies in the LG and Aquarius samples.

summarizes the main properties of the $\mathrm{LG}$ and Aquarius galaxies at $z=0$ inside their respective virial radii $R_{200}$, where the density is 200 times the critical density $\rho_{c}$ at the corresponding redshift.

The LG galaxies have stellar components that all show some measure of rotational support; however, when we examine the components of angular momenta (e.g., as in S09) we see that only half, G2, G3, and G4, have a significant fraction $(>17 \%)$ of the stellar mass in a rotationally supported disk. Nevertheless, all these galaxies exhibit extended gas disks and star formation at redshift zero. In the case of the Aquarius simulations, each galaxy was able to grow extended disks during their evolution, but again only half can survive until $z=0$, Aq-C, Aq-D, Aq-E, and Aq-G. In both cases, we find the survival or destruction of disks in the S09 sample was found to depend primarily on the occurence of major mergers and the alignment between the angular momenta of the stars and gas in the inner regions (see also Scannapieco et al. 2015).

\section{ENVIRONMENTAL EFFECTS}

The constrained nature of our simulation allows us to explore the possibility that environment plays a role in the determination of the properties of galaxies like our MW. In this section, we investigate this by comparing the properties of the LG and Aquarius galaxies. We compare properties that are expected to depend on environment (gas mass, stellar mass, SFR) to a measure of the environment. We note that the number of galaxies in our samples is too small to determine quantitative trends, but that systematic differences may still be visible within the sample.

For our environmental measure we use the ratio of the mean density of matter within $1200 \mathrm{ckpc}$ to the mean density of matter of the universe, i.e.,

$$
\delta_{1200} \equiv \frac{\left\langle\rho_{M}\right\rangle_{r<1200 \mathrm{ckpc}}}{\Omega_{M}(z) \rho_{c}(z)} .
$$

To give some physical reference, this radius corresponds to how far a baryon traveling at $100 \mathrm{~km} \mathrm{~s}^{-1}$ traverses in $12 \mathrm{Gyr}$ (ignoring cosmological expansion), approximately identifying 

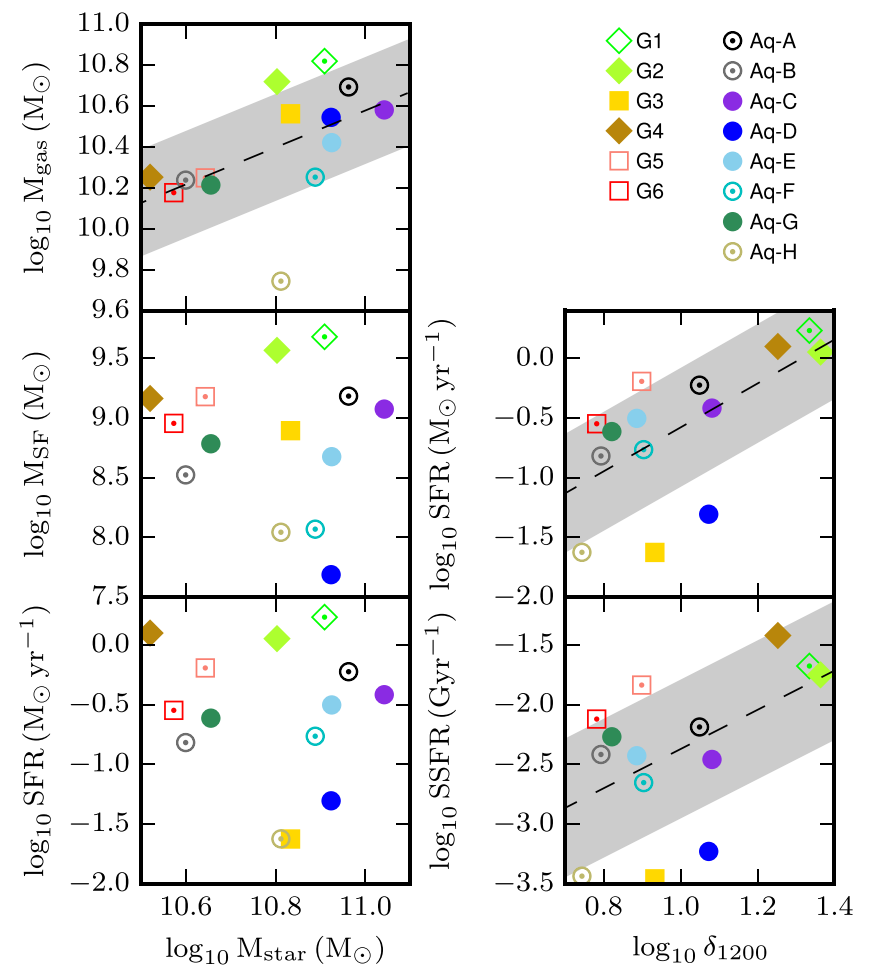

Figure 3. Comparison between the properties of the galaxy samples at $z=0$. Left column from top to bottom shows the gas mass $\left(M_{\text {gas }}\right)$, the star-forming gas mass $\left(M_{\mathrm{SF}}\right)$, and the star formation rate (SFR), all as a function of stellar mass $\left(M_{\text {star }}\right)$. Right column shows the SFR and the specific star formation rate (SSFR) with $\delta_{1200}$, respectively. Filled symbols indicate the galaxies that have stellar disk, diamonds are those in the rich sample, and gray-shaded regions, where present, indicate the significant linear regressions with $\pm 1 \sigma$ errors.

the cosmological neighborhood which can affect a MW-mass halo. This scale was also used as proxy for the "local volume" by Garrison-Kimmel et al. (2014), and, in the case of the LG simulation, is large enough to include the halos of both G1 and $\mathrm{G} 2$ at the present time, but for all other galaxies forms disjoint volumes. We have considered scales from 600 to $1500 \mathrm{ckpc}$ and found this to give the least stochastic results.

Figure 2 compares the environments of our galaxies as a function of redshift, giving us a measure of the environmental "assembly," although we would stress that at these large radii we are not seeing halo mergers but rather a growth in the richness of the environment. Apparent is that although all the galaxies inhabit similar environments at $z \sim 2-3$, the three galaxies G1, G2, and G4 exhibit stronger evolution after $z \approx 2$ and are the galaxies with the highest overdensity $\delta_{1200}$ at $z=0$, approximately twice as overdense as the Aquarius galaxies and the remaining LG galaxies. Additionally, these three all inhabit gaseous filaments, the former two reside in the filament identified by Nuza et al. (2014b), and the latter can be seen in the animation associated with Figure 1. Such structures have previously been implicated as affecting SF in groups (Bahé et al. 2013). Since these three galaxies recur as outliers later, we denote them the "rich sample" and by contrast the remaining 11 as the "poor sample." We note that this evolution of $\delta_{1200}$ implies imprints due to environment on the rich sample will disappear at early epochs $(z \gtrsim 1)$, before they were outliers.

In the left-hand column of Figure 3 we compare the distribution of three fundamental properties of the galaxies at $z=0$ : the gas mass $\left(M_{\mathrm{gas}}\right)$ and the star-forming gas mass $\left(M_{\mathrm{SF}}\right)$

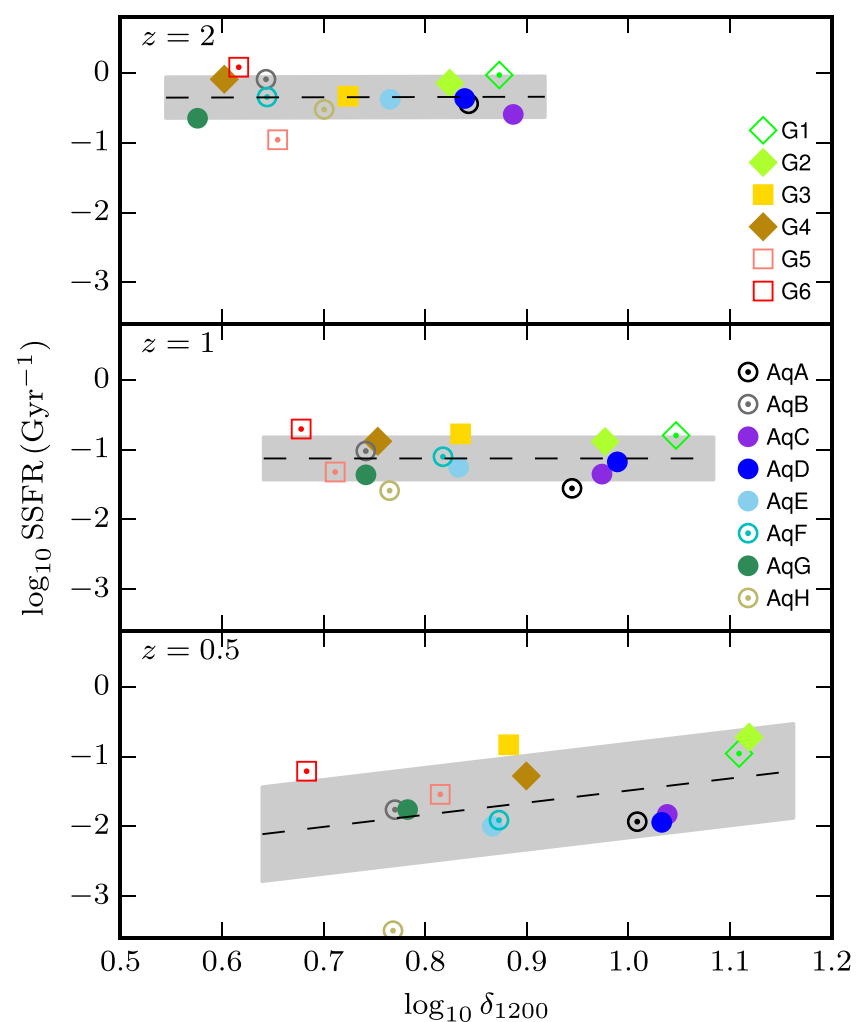

Figure 4. Same as for the lower right panel of Figure 3, but for redshifts $z=2$, $1,0.5$ from top to bottom.

within $R_{200}$, and the SFR for material within $30 \mathrm{kpc}$ averaged over the last $500 \mathrm{Myr}$. These quantities are all plotted as a function of the present-day stellar masses $\left(M_{\text {star }}\right)$ and we additionally mark the galaxies with stellar disks.

The cosmological trend of $M_{\text {gas }}$ to rise with $M_{\text {star }}$ is visible even within this restricted stellar mass sample, and indeed the significance of the trend shown is $4.4 \sigma$. For $M_{\mathrm{SF}}$ and SFR the trends are insignificant, partly due to the higher stochasticity of these quantities (note the dynamic range of $M_{\mathrm{SF}}$ and the SFR is much higher than just for the gas) that obscures the trend in such a limited sample, both in terms of small number and restricted stellar mass range. More interestingly, perhaps, is that two of the rich sample (G1 and G2) are the outliers in $M_{\mathrm{gas}}$, $M_{\mathrm{SF}}$ and SFR, and if we only consider SFR then the entire rich sample is extremal. This suggests the possibility that the richer environments of these galaxies are contributing to higher SFRs than those found in the poor sample. There does not appear to be corresponding trends with the morphology (i.e., whether the stellar component lies in a disk) as was also noted by Few et al. (2012), and indeed in Scannapieco et al. (2015) we find morphology to be largely determined by the merger history.

These hints of an effect of the denser environment are borne out by classification with environmental overdensity (Figure 3 right-hand side). The SFR versus overdensity has significant correlation, rejecting the null hypothesis at $3.8 \sigma$. To account for comorbidity of $\delta_{1200}$ with $M_{\text {star }}$, we additionally considered the specific star formation rate (SSFR, for which we simply use SFR $\left.M_{\text {star }}\right)$ and $M_{\text {star }}$ versus $\delta_{1200}$ (not shown). The significance of the SSFR trend is lower $(2.6 \sigma)$, though both are more significant than the correlation of $\delta_{1200}-M_{\text {star }}(0.8 \sigma)$, i.e., environment is a better indicator of SFR than stellar mass. The 
correlation between $M_{200}$ and SFR is also poor as the virial mass closely traces $M_{\text {star }}$.

Having seen an effect of environment on SFR for MW-mass galaxies at $z=0$, it is interesting to consider whether this exists for their progenitors at higher redshifts. Our simulations also provide evidence here, although we must be somewhat cautious as galaxies are correlated with their progenitors. In Figure 4 we plot the SSFR versus $\delta_{1200}$ for our 14 galaxies at $z=2$, 1 , and 0.5 . At higher redshifts, the galaxies live in sparser environments with higher SSFRs, and noticeably the $\delta_{1200}$-SSFR correlation fades toward earlier epochs.

Comparing Figures $2-4$, we can see that from $z \lesssim 1$ the rich sample transitions to a denser environment with a modest drop in SSFR while the poor sample has a more pronounced fall in SSFR but less evolution in $\delta_{1200}$. This suggests a possible explanation for the trends in Figure 3: the cold gas is driven by the richness of the environment, i.e., the accretion of dense structures allows the star forming gas to replenish and higher rates of star formation to continue to $z=0$. In our simulations subhalos deposit little of this due to their low gas content (Nuza et al. 2014b), although they may trigger star formation indirectly. Intriguingly, our trends for SFRs and star-forming gas masses are in opposition to that of clusters versus the field, where suppression extends to group scales (e.g., McGee et al. 2008 though see also Ziparo et al. 2013). This may result from the density of those systems compared to our sample, and in the case of the LG galaxies from the fact that the LG has a late assembly, and so environmental effects will be less pronounced compared to more evolved systems.

\section{CONCLUSIONS}

In this Letter we performed a hydrodynamical constrained simulation of the structures within $10 \mathrm{Mpc}$ of the MW to search for megaparsec-scale environmental effects on galaxy properties. We compare the SFRs, morphologies and gas fractions of six galaxies of MW-like stellar masses to eight from unconstrained simulations and find systematically higher SFRs in the galaxies that live in richer environments, though this trend did not extend to morphologies.

Most notably, the simulated MW and M31 candidates are overabundant both in present-day SSFR and in environmental density, marking them as outliers compared to the remaining galaxies. These exceptional values disappear at earlier cosmological times, corresponding to their environmental assembly. The third galaxy which displayed this trend inhabited the dense environment of a filamentary structure.

Although the number of galaxies is small and larger samples are needed to confirm these trends, our results suggest that galaxies of a given mass that live in richer environments could more easily replenish their gas reservoirs enabling higher SFRs. This is consistent with a picture where the morphologies of MW-like galaxies are primarily set by their merger history, yet environment still plays a role in their star formation histories. This demonstrates the need to understand in detail the effects of environment on galaxy properties if we are to understand the formation of our own galaxy.
The simulation was performed on the MareNostrum cluster in Barcelona. C.S. and P.C. acknowledge support from the Leibniz Gemeinschaft through grant SAW-2012-AIP-5 129. S.E.N. acknowledges support from the Deutsche Forschungsgemeinschaft under the grants MU 1020 16-1 and NU 332/2-1, and G.Y. thanks MINECO (Spain) for supporting his research through different projects: AYA2012-31101, FPA2012-34694 and Consolider Ingenio SyeC CSD2007-0050.

\section{REFERENCES}

Aumer, M., White, S. D. M., Naab, T., \& Scannapieco, C. 2013, MNRAS, 434, 3142

Bahé, Y. M., McCarthy, I. G., Balogh, M. L., \& Font, A. S. 2013, MNRAS, 430, 3017

Blanton, M. R., Eisenstein, D. J., Hogg, D. W., et al. 2003, BAAS, 203, $\# 145.01$

Coenda, V., Muriel, H., \& Martínez, H. J. 2014, arXiv:1410.7780

Creasey, P., Theuns, T., Bower, R. G., \& Lacey, C. G. 2011, MNRAS, 415,3706

Darg, D. W., Kaviraj, S., Lintott, C. J., et al. 2010, MNRAS, 401, 1043

de Ravel, L., le Fèvre, O., Tresse, L., et al. 2009, A\&A, 498, 379

Dressler, A. 1980, ApJ, 236, 351

Ellison, S. L., Patton, D. R., Simard, L., et al. 2010, MNRAS, 407, 1514

Fakhouri, O., Ma, C.-P., \& Boylan-Kolchin, M. 2010, MNRAS, 406, 2267

Few, C. G., Gibson, B. K., Courty, S., et al. 2012, A\&A, 547, A63

Garrison-Kimmel, S., Boylan-Kolchin, M., Bullock, J. S., \& Lee, K. 2014, MNRAS, 438, 2578

Girardi, M., Rigoni, E., Mardirossian, F., \& Mezzetti, M. 2003, A\&A, 406, 403

Gottlöber, S., Hoffman, Y., \& Yepes, G. 2010, arXiv:1005.2687

Guedes, J., Callegari, S., Madau, P., \& Mayer, L. 2011, ApJ, 742, 76

Guzzo, L., Strauss, M. A., Fisher, K. B., Giovanelli, R., \& Haynes, M. P. 1997, ApJ, 489, 37

Haardt, F., \& Madau, P. 1996, ApJ, 461, 20

Hermit, S., Santiago, B. X., Lahav, O., et al. 1996, MNRAS, 283, 709

Hess, K. M., \& Wilcots, E. M. 2013, AJ, 146, 124

Jian, H.-Y., Lin, L., \& Chiueh, T. 2012, ApJ, 754, 26

Katz, N., \& White, S. D. M. 1993, ApJ, 412, 455

Knebe, A., Libeskind, N. I., Knollmann, S. R., et al. 2011, MNRAS, 412, 529

Lewis, I., Balogh, M., De Propis, R., et al. 2002, MNRAS, 334, 673

Lin, L., Cooper, M. C., Jian, H.-Y., et al. 2010, ApJ, 718, 1158

Maulbetsch, C., Avila-Reese, V., Colín, P., et al. 2007, ApJ, 654, 53

McGee, S. L., Balogh, M. L., Henderson, R. D. E., et al. 2008, MNRAS, 387,1605

Nuza, S. E., Kitaura, F.-S., Heß, S., Libeskind, N. I., \& Müller, V. 2014, MNRAS, 445, 988

Nuza, S. E., Parisi, F., Scannapieco, C., et al. 2014, MNRAS, 441, 2593

Scannapieco, C., Creasey, P., Nuza, S. E., et al. 2015, A\&A, submitted

Scannapieco, C., Gadotti, D. A., Jonsson, P., \& White, S. D. M. 2010, MNRAS, 407, L41

Scannapieco, C., Tissera, P. B., White, S. D. M., \& Springel, V. 2005, MNRAS, 364, 552

Scannapieco, C., Tissera, P. B., White, S. D. M., \& Springel, V. 2006, MNRAS, 371, 1125

Scannapieco, C., Tissera, P. B., White, S. D. M., \& Springel, V. 2008, MNRAS, 389, 1137

Scannapieco, C., White, S. D. M., Springel, V., \& Tissera, P. B. 2009, MNRAS, 396, 696

Scannapieco, C., White, S. D. M., Springel, V., \& Tissera, P. B. 2011, MNRAS, 417, 154

Scannapieco, C., Wadepuhl, M., Parry, O. H., et al. 2012, MNRAS, 423, 1726

Springel, V., Wang, J., Vogelsberger, M., et al. 2008, MNRAS, 391, 1685

Stinson, G. S., Bovy, J., Rix, H.-W., et al. 2013, MNRAS, 436, 625

Tonnesen, S., \& Cen, R. 2012, MNRAS, 425, 2313

Toomre, A., \& Toomre, J. 1972, ApJ, 178, 623

Vogelsberger, M., Genel, S., Springel, V., et al. 2014, MNRAS, 444, 1518

Yepes, G., Gottlöber, S., \& Hoffman, Y. 2014, NewAR, 58, 1

Ziparo, F., Popesso, P., Biviano, A., et al. 2013, MNRAS, 434, 3089 